\title{
Some Prototype Results of the Symmetric Regularized Long Wave Equation Arising in Nonlinear Ion Acoustic Waves
}

\author{
Erdem IŞIK ${ }^{1}$, Hasan BULUT ${ }^{2}$, Sibel Şehriban ATAŞ, ${ }^{2 *}$ \\ ${ }^{1}$ Tunceli Vocational School, Munzur University, Tunceli, Turkey \\ ${ }^{2}$ Faculty of Science, Department of Mathematics, Firat University, Elazig, Turkey
}

\begin{abstract}
In this manuscript, we consider the Bernoulli sub-equation function method for obtaining new exponential prototype structures to th Symmetric Regularized Long Wave mathematical model. We obtain new results by using this technique. We plot two- and three-dimensional surfaces of the results by using Wolfram Mathematica 9. At the end of this manuscript, we submit a conclusion in the comprehensive manner.
\end{abstract}

\section{Introduction}

In the recent years, the investigation of exact travelling wave solutions to nonlinear partial differential equations plays an important role in the study of nonlinear modelling physical phenomena. Also the study of the travelling wave solutions plays an important role in nonlinear science. A variety of powerful methods has been presented, such as Hirota's bilinear method[1]. the inverse scattering transform [2], sine-cosine method [3], homotopy analysis method [4,5], variational iteration method [6-8]. We study different aspects of the Symmetric Regularized-Long-Wave equation (SRLW). The Symmetric Regularized-LongWave equation is a model for the weakly nonlinear ion acoustic and space-charge waves[9]. This equation was introduced by Seyler and Fenstermacher in[10]. Moreover, some important results have been submitted to the literature by various scientists [11,21]

The paper is organized as follows: In section 2, we describe the Bernoulli SubEquation function method (BSEFM). In section 3, we consider the following Symmetric

*Corresponding author: suzundag90@gmail.com 
Regularized Long Wave equation [22] defined by

$$
u_{t t}+u_{x x}+u u_{x t}+u_{x} u_{t}+u_{x x t t}=0 \text {. }
$$

Moreover, in section 4 we give the physical interpretations and remarks of the solutions obtained by BSEFM. Also a comprehensive conclusion is given in section 5 .

\section{Fundamental Properties of the Method}

An approach to the mathematical models including partial differential equations will be presented in this sub-section of paper. The steps of the this technique which is partially new modified can be taken as follows [10].

Step 1. We consider the following nonlinear partial differential equation (NLPDE) in two variables and a dependent variable $u$;

$$
P\left(u_{x}, u_{t}, u_{x t}, u_{x x}, \cdots\right)=0 \text {, }
$$

and take the travelling wave transformation

$$
u(x, t)=U(\eta), \eta=x-c t,
$$

where $c \neq 0$. Substituting Eq.(3) in Eq.(2) yields a nonlinear ordinary differential equation (NLODE) as following;

$$
N\left(U, U^{\prime}, U^{\prime \prime}, \cdots\right)=0
$$

where $\quad U=U(\eta), U^{\prime}=\frac{d U}{d \eta}, U^{\prime \prime}=\frac{d^{2} U}{d \eta^{2}}, \cdots$.

Step 2. Take trial equation of solution for Eq.(4) as following:

$$
U(\eta)=\sum_{i=0}^{n} a_{i} F^{i}=a_{0}+a_{1} F+a_{2} F^{2}+\cdots+a_{n} F^{n},
$$

and

$$
F^{\prime}=b F+d F^{M}, b \neq 0, d \neq 0, M \in \mathbb{R}-\{0,1,2\},
$$

where $F(\eta)$ is Bernoulli differential polynomial. Substituting Eq.(5) along with Eq.(6) in Eq.(4), it yields an equation of polynomial $\Omega(F(\eta))$ of $F(\eta)$ as following;

$$
\Omega(F(\eta))=\rho_{s} F(\eta)^{s}+\cdots+\rho_{1} F(\eta)+\rho_{0}=0 .
$$

According to the balance principle, we can obtain a relationship between $n$ and $M$.

Step 3. Let the coefficients of $\Omega(F(\eta))$ all be zero will yield an algebraic equations system;

$$
\rho_{i}=0, i=0, \cdots, s .
$$

Solving this system, we will specify the values of $a_{0}, \cdots, a_{n}$.

Step 4. When we solve Eq.(6), we obtain the following two situation according to $b$ and d; 


$$
\begin{gathered}
F(\eta)=\left[\frac{-d}{b}+\frac{E}{e^{b(M-1) \eta}}\right]^{\frac{1}{1-M}}, b \neq d, \\
F(\eta)=\left[\frac{(E-1)+(E+1) \tanh \left(\frac{b(1-M) \eta}{2}\right)}{1-\tanh \left(\frac{b(1-M) \eta}{2}\right)}\right]^{\frac{1}{1-M}}, b=d, \quad E \in \mathbb{R} .
\end{gathered}
$$

Using a complete discrimination system for polynomial, we obtain the solutions to Eq.(4) with the help of Wolfram Mathematica 9 programming and classify the exact solutions to Eq.(4). For a better understanding of results obtained in this way, we can plot two- and three-dimensional surfaces of solutions by taking into consideration suitable values of parameters.

\section{Application}

When we consider the travelling wave transformation and perform the transformation $u(x, t)=U(\eta), \eta=k x-c t$ which $c$ is constant non-zero in Eq.(1), we obtain NLODE as following;

$$
2\left(k^{2}+c^{2}\right) U-c k U^{2}+c^{2} k^{2} U^{\prime \prime}=0 .
$$

When we consider to Eq.(5) and Eq.(6) for balance principle between $U^{\prime \prime}$ and $U^{2}$, we obtain the following relationship between $n$ and $M$;

$$
2 M=n+2 .
$$

This relationship gives us some new different complex travelling wave solutions for Eq.(1).

Case 1: If we take as $n=4$ and $M=3$ in Eq. (11), we can write following equations;

$$
\begin{aligned}
& U=a_{0}+a_{1} F+a_{2} F^{2}+a_{3} F^{3}+a_{4} F^{4}, \\
& U^{\prime}=a_{1} b F+a_{1} d F^{3}+2 a_{2} b F^{2}+2 a_{2} d F^{4}+3 a_{2} b F^{3}+ \\
& +3 a_{3} d F^{5}+4 a_{4} b F^{4}+4 a_{4} d F^{6}
\end{aligned}
$$

and

$$
\begin{aligned}
& U^{\prime \prime}=b^{2} F a_{1}+4 b d F^{3} a_{1}+3 d^{2} F^{5} a_{1}+4 b^{2} F^{2} a_{2}+12 b d F^{4} a_{2}+ \\
& +8 d^{2} F^{6} a_{2}+9 b^{2} F^{3} a_{3}+24 b d F^{5} a_{3}+15 d^{2} F^{7} a_{3}+16 b^{2} F^{4} a_{4}+ \\
& +40 b d F^{6} a_{4}+24 d^{2} F^{8} a_{4} \\
& \vdots
\end{aligned}
$$

where $a_{2} \neq 0, b \neq 0, d \neq 0$. When we put Eq.(12-14) in Eq.(10), we obtain a system of algebraic equations for Eq.(13). Therefore, we obtain a system of algebraic equations from 
these coefficients of polynomial of $F$. Solving this system with the help of wolfram Mathematica 9, we find the following coefficients and solutions;

Case 1a. For $b \neq d$, it can be considered the following coefficients;

$$
\begin{aligned}
& a_{1}=0, a_{2}=\frac{24 \sqrt{2} d k a_{0}}{\sqrt{a_{0}\left(a_{0}-\sqrt{-16+a_{0}^{2}}\right)}}, a_{3}=0, \\
& a_{4}=12 d^{2} k^{2}\left(a_{0}+\sqrt{-16+a_{0}^{2}}\right), \\
& c=\frac{1}{4} k\left(a_{0}+\sqrt{-16+a_{0}^{2}}\right), b=\frac{\sqrt{a_{0}\left(a_{0}-\sqrt{-16+a_{0}^{2}}\right)}}{4 \sqrt{2 k}} .
\end{aligned}
$$

Substituting these coefficients in Eq.(12) along with Eq.(9a), we obtain the following new exponential function solution for Eq.(1);

$$
u_{1}(x, t)=a_{0}+\frac{24 a_{0} d \sqrt{2} e^{\frac{\rho\left(-4 x+t\left(a_{0}+\sqrt{-16+a_{0}^{2}}\right)\right)}{8 \sqrt{2}}} E k \rho}{\left(-4 \sqrt{2} d k+e^{\varpi\left(-4 x+t\left(a_{0}+\sqrt{-16+a_{0}^{2}}\right)\right)} E \rho\right)^{2}},
$$

where $a_{0}, d, k$ are real constants and $\rho=\sqrt{a_{0}\left(a_{0}-\sqrt{-16+a_{0}^{2}}\right)}, \varpi=\frac{\rho}{8 \sqrt{2}}$.
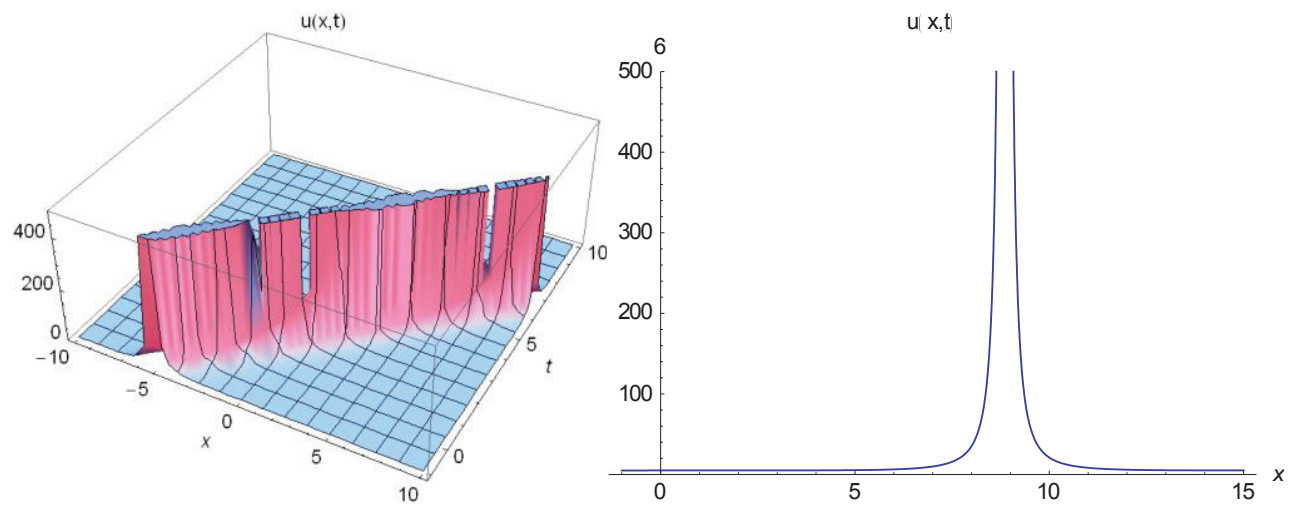

Figure 1. The 2D and 3D surfaces of Eq.(16) under the values of $a_{0}=5, k=0.3, d=50, E=0.8,-10<x<10,-1<t<10$ and $t=6$ for 2D surfaces. 
Case 1b. Another coefficients for Eq.(1) and for $b \neq d$, it can be considered follows;

$$
\begin{aligned}
& a_{0}=\frac{2 c}{k}+\frac{2 k}{c}, a_{1}=0, a_{2}=-24 d \sqrt{c^{2}+k^{2}}, a_{3}=0, \\
& a_{4}=48 c d^{2} k, b=-\frac{\sqrt{c^{2}+k^{2}}}{2 c k} .
\end{aligned}
$$

When we regulate Eq.(12) under the terms of Eq.(9a,17), we find the other new exponential function solution for Eq.(1);

$$
u_{2}(x, t)=\frac{2 c}{k}+\frac{2 k}{c}+\frac{48 c d^{2} k}{\left(e^{v(-c t+k x)} E+\frac{2 d}{v}\right)^{2}}-\frac{24 d v c k}{2 c d k+v c k e^{v(-c t+k x)} E}
$$

where $c, d, k$ are real constants and $v=\frac{\sqrt{c^{2}+k^{2}}}{c k}$.
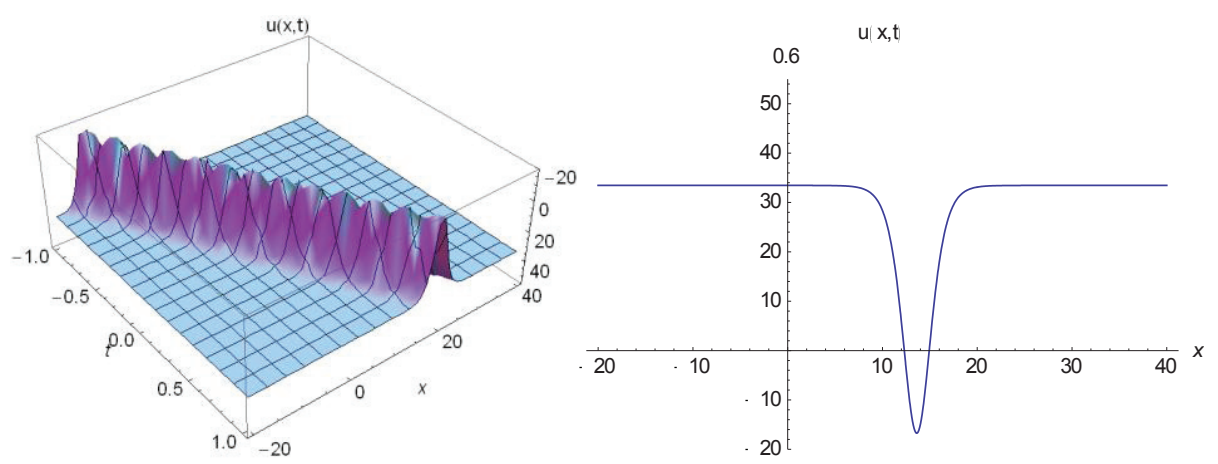

Figure 2. The 2D and 3D surfaces of Eq.(18) under the values of $c=5, k=0.3, d=50, E=0.8,-20<x<40,-1<t<1, t=0.6$ for 2D surfaces.

Case 1c. Another coefficients to the Eq.(1) for $b \neq d$, it can be considered as follows;

$$
\begin{aligned}
& a_{0}=\frac{8 b^{2} c^{2}}{\sqrt{-1+4 b^{2} c^{2}}}, a_{1}=0, a_{2}=\frac{48 b c^{2} d}{\sqrt{-1+4 b^{2} c^{2}}}, a_{3}=0, \\
& a_{4}=\frac{48 c^{2} d^{2}}{\sqrt{-1+4 b^{2} c^{2}}}, k=\frac{c}{\sqrt{-1+4 b^{2} c^{2}}} .
\end{aligned}
$$

Considering these coefficients in Eq.(12) along with Eq.(9a,19), we gain other new complex exponential function solution to Eq.(1); 


$$
u_{3}(x, t)=\frac{8 b^{2} c^{2}\left(d^{2} e^{4 b c(-t+\kappa x)}+4 b d e^{2 b c(-t+\kappa x)} E+b^{2} E\right)}{\sqrt{-1+4 b^{2} c^{2}}\left(d e^{2 b c(-t+\kappa x)}-b E\right)^{2}}
$$

where $b, c, d$ are real constants and $\kappa=\frac{1}{\sqrt{-1+4 b^{2} c^{2}}}$.
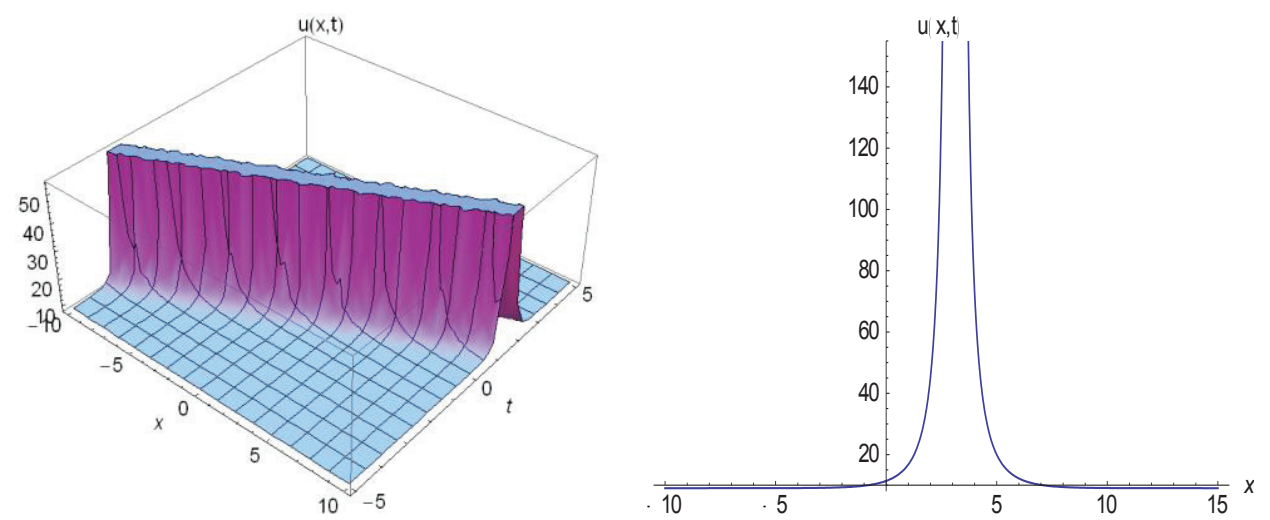

Figure 3. The 2D and 3D surfaces of real part of Eq.(20) under the values of $b=2, d=0.1, c=1.1, E=0.8,-10<x<10,-5<t<5, t=0.1$ for $2 \mathrm{D}$ surfaces.

\section{DISCUSSION AND CONCLUSIONS}

The solutions such as Eq.(16,18,20) obtained by using BSEFM are the new exponential function structures solutions for Eq.(1) when we compare with the paper submitted to the literature by [22]. It has been observed that all solutions have verified the Eq. (1) by using Wolfram Mathematica 9. To the best of our knowledge, the application of BSEFM to the Eq.(1) has not been previously submitted to the literature. The method proposed in this paper can be used to seek more travelling wave solutions of NLEEs because the method has some advantages such as easily calculations, writing programme for obtaining coefficients and so many. Eq.(18) can be rewritten as hyperbolic functions by using the fundamental properties of hyperbolic functions as follows;

$u_{1}(x, t)=\frac{2 c}{k}+\frac{2 k}{c}+\frac{48 c d^{2} k}{\left(E e^{v(-c t+k x)}+\frac{2 d}{v}\right)^{2}}-\frac{24 d\left(c^{2}+k^{2}\right)}{2 c d k+E v k c e^{v(-c t+k x)}}$,

where $c, d, k$ are real constants and $v=\frac{\sqrt{c^{2}+k^{2}}}{c k}$. When we consider the general properties of hyperbolic and exponential functions, we can rewrite $u_{1}(x, t)$ solution in the form of hyperbolic function as following. 


$$
\begin{aligned}
& u_{1}(x, t)=\frac{2 c}{k}+\frac{2 k}{c}+\frac{48 c d^{2} k}{\left(\frac{E \pm E \sqrt{1-\sec h^{2}(T)}}{\sec h(T)}+\delta\right)^{2}}-\frac{24 d\left(c^{2}+k^{2}\right)}{2 c d k+\frac{\rho \pm \rho \sqrt{1-\sec h^{2}(T)}}{\operatorname{sech}(T)}} \\
& T=\frac{(-c t+k x)}{c k} \sqrt{c^{2}+k^{2}}, \delta=\frac{2 c d k}{\sqrt{c^{2}+k^{2}}}, \rho=E \sqrt{c^{2}+k^{2}} .
\end{aligned}
$$

\section{References}

1. R. Hirota, The Direct Method in Soliton Theory. Cambridge Univ. Press, (2004).

2. M.J. Ablowitz, P.A. Clarkson, Solitons, nonlinear evolution equations and inverse scattering, Cambridge: Cambridge University Press, (1991).

3. A. M. Wazwaz, Travelling wave solutions for combined and double combined sinecosineGordon equations by the variable separated ODE method. Appl. Math. Comput, 177, 755-760 (2006).

4. M. Dehghan, J. Manafian, A. Saadatmandi, The solution of the linear fractional partial differential equations using the homotopy analysis method. Z. Naturforsch, 65a, 935949 (2010).

5. M. Dehghan, J. Manafian, A. Saadatmandi, Solving nonlinear fractional partial differential equations using the homotopy analysis method. Num. Meth. Partial Differential Eq., 26, 448-479 (2010).

6. J. H. He, Variational iteration method a kind of non-linear analytical technique: some examples. Int. J. Nonlinear Mech, 34, 699-708 (1999).

7. M. Dehghan, M. Tatari, Identifying an unknown function in a parabolic equation with overspecified data via He's variational iteration method. Chaos Solitons Fractals, 36, 157-166 (2008).

8. M. Dehghan, J. Manafian, A. Saadatmandi, Application of semi-analytic methods for the Fitzhugh-Nagumo equation, which models the transmission of nerve impulses, Math. Meth. Appl. Sci, 33, 1384-1398 (2010).

9. Carlos Banquet Brango, The Symmetric Regularized-Long-Wave equation: Wellposedness and nonlinear stability. Physica, D(241), 125-133 (2012).

10. C. Seyler, D. Fenstermacher, A symmetric regularized-long-wave equation. Phys. Fluids 27, 4-7 (1984). 
11. G. K. Watugala, Sumudu Transform: A New Integral Transform to Solve Differantial Equations and Control Engineering Problems. International Journal of Mathematical Education in Science and Technology, 24, 35-43 (1993).

12. Y. Pandir, New exact solutions of the generalized Zakharov-Kuznetsov modified equal-width equation. Pramana journal of physics, 82(6), 949-964 (2014).

13. H. Bulut, H. M. Baskonus and F. B. M. Belgacem, The Analytical Solutions of Some Fractional Ordinary Differential Equations by Sumudu Transform Method. Abstract and Applied Analysis,(2013).

14. A. M. Wazwaz, The tanh method: solitons and periodic solutions for Dodd-BulloughMikhailov and Tzitzeica- Dodd-Bullough equations. Chaos, Solitons and Fractals, 25, 55-56 (2005).

15. C.S. Liu, A new trial equation method and its applications. Communications in Theoretical Physics , 45(3), 395-397 (2006).

16. C.S. Liu, Trial Equation Method to Nonlinear Evolution Equations with Rank Inhomogeneous: Mathematical Discussions and Its Applications. Communications in Theoretical Physics, 45(2), 219-223.

17. H. Bulut, Y. Pandir, H. M. Baskonus, Symmetrical Hyperbolic Fibonacci Function Solutions of Generalized Fisher Equation with Fractional Order. AIP Conf., 1558, 1914 (2013).

18. Y. Pandir, Y. Gurefe, U, Kadak, and E. Misirli, Classifications of exact solutions for some nonlinear partial differential equations with generalized evolution. Abstract and Applied Analysis, 2012, 16 pages, (2012).

19. Ryabov, P. N., Sinelshchikov, D. I., and Kochanov, M. B., Application of the Kudryashov method for finding exact solutions of the high order nonlinear evolution equations. Applied Mathematics and Computation, 218(7), 3965-3972 (2011).

20. Kudryashov, N. A., One method for finding exact solutions of nonlinear differential equations. Communications in Nonlinear Science and Numerical Simulation, 17(6), 2248-2253 (2012).

21. Lee J., and Sakthivel, R., Exact travelling wave solutions for some important nonlinear physical models. Pramana-Journal of Physics, 80(5), 757-769 (2013).

22. H. Bulut, H.M.Baskonus, E. Cuvelek, On The Prototype Solutions of Symmetric Regularized Long Wave Equation by Generalized Kudryashov Method. Mathematics letters, 1(2), 10-16 (2015). 